\title{
Damping function in the penetration/perforation dynamics of rigid projectiles
}

\author{
X. W. Chen, X. L. Li \& K. W. Deng \\ Institute of Structural Mechanics, \\ China Academy of Engineering Physics, P.O. Box 919-414, \\ Mianyang City, Sichuan Province 621900, China
}

\begin{abstract}
This paper defines a third dimensionless parameter, i.e., the damping function, besides the impact function and geometry function of a projectile introduced by the author previously, in the penetration/perforation dynamics of a rigid projectile. It only depends on the interaction of projectile and target materials and is independent of projectile geometry. A general penetration resistance, which contains the terms of viscous effect and the dummy mass of a projectile induced by the deceleration effect, is adopted in the formulation. A dimensionless formula of depth of penetration is conducted with only these three parameters for general convex shapes of various rigid projectiles. Accounting for the influence of the damping function, the normal perforations of thick metallic plates struck by sharp nose rigid projectiles are studied further.

Keywords: penetration, perforation, rigid projectile, damping function, metal, concrete, ceramics.
\end{abstract}

\section{Introduction}

The experimental method is still one of the major means of penetration investigation. Usually, the empirical formulae are proposed by fitting normalization of a large amount of experimental data of different reduced or same scale firing tests, and are further applied to the prediction of other similar penetration problems. The Poncelet equation [1], i.e., $F=A_{0}\left(a+b V^{2}\right)$, is the most venerable and classical empirical formula used to calculate the penetration 
resistance of metallic targets stricken by a flat-nosed projectile, where $A_{0}$ is a cross-section area of the projectile, $V$ is the impact velocity, and $a$ and $b$ are two material constants to be determined by experiments. However, after accounting for the geometry of the projectile, the dynamic cavity expansion model with simple elastic-plastic constitute of target material, further developed by Forrestal and his colleagues, presents the same form and thus offers a theoretical foundation of the Poncelet equation. The penetration resistance consists of two terms, i.e., the static strength term of the target material and the flow stress term (related to $V^{2}$ ). Based on the dynamic cavity expansion theory, Chen and $\mathrm{Li}$ [2] and $\mathrm{Li}$ and Chen [3] indicate that only two dimensionless numbers, i.e., the impact function I and the geometry function of projectile $\mathrm{N}$ dominate in the penetration of rigid projectiles.

More commonly, due to the diversification of target materials, the dynamic cavity expansion model has been further employed to deep penetrations of rock, soil and ceramics as well as to metal and concrete [4-8]. Penetration resistance, which has been recently employed in concrete and ceramic mediums, includes not only the above mentioned two terms, but also the damping effect of target material (related to $V$ ). In particular, the damping effect on the penetration resistance may become remarkable when polymer or cellular alloys are being used as the target material. Meanwhile, accompanied with the improvement of projectile material in the development of deep penetration warheads, the upper velocity limit of rigid projectiles has been improved from $900-1000 \mathrm{~m} / \mathrm{s}$ to $1700-1800 \mathrm{~m} / \mathrm{s}$. The effect of the dummy mass caused by the higher velocity impact becomes more and more distinct, while it is always omitted in the formulation of dynamic cavity expansion theory because of mathematical simplification. Therefore, the expression of penetration resistance should further take account of the damping effect of the target material and dummy mass, i.e., $\sigma=a+b v+c v^{2}+d \dot{v}$, where $a, b, c$ and $d$ are all material coefficients. The above equation is usually regarded as the alternative general formula of penetration resistance. Accompanied with the introduction of various target mediums, more and more attention has recently been paid to the application of the general formula of penetration resistance in penetration dynamics.

In this paper, a third dimensionless parameter, i.e., the damping function $\xi$, is defined in the penetration dynamics of rigid projectiles besides the impact function $I$ and the geometry function of projectile $N$ introduced by the author previously [9]. The dimensionless formula of DOP is conducted in the expression of $I, N$ and $\xi$ for general convex shapes of various rigid projectiles.

\section{General expression of penetration resistance}

Penetration resistance is always expressed as the integral form of normal stress on the nose surface that may be solved according to the dynamic cavity expansion theory. A non-deformable projectile having a common convex nose shape, as shown in Fig.1, impacts a target normally at velocity $V_{0}$ and proceeds to penetrate the target medium at rigid-body velocity $V$. The dynamic cavity 
expansion analysis yields a general relationship between the normal compressive stress $\sigma_{n}$ on the projectile nose and the normal expansion velocity $v$ [10],

$$
\sigma_{n}=A Y+C \sqrt{\rho Y} \cdot v+B \rho v^{2}+D \rho d \cdot \dot{v}
$$

where $Y$ and $\rho$ are yielding stress and density of the target material, respectively. Distinguished from previous definition, $d$ is the shank diameter of the projectile in eqn (1) and the following formulations. $A, B, C$ and $D$ are dimensionless material constants of target. The second term on the right-hand side of eqn (1) describes the damping effect and the final term introduces the influence of dummy mass induced by the deceleration effect.

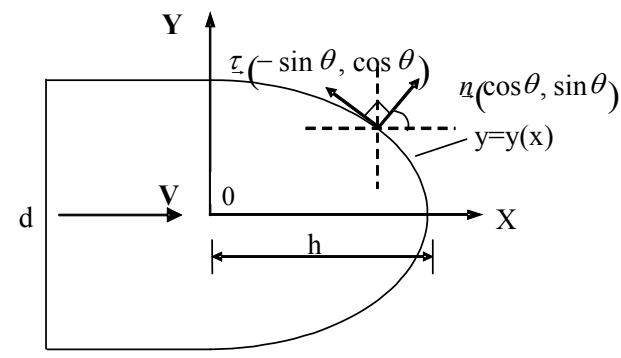

Figure 1: General nose shape of a rigid projectile.

The resulting axial resistant force on the projectile nose can be integrated as

$$
F_{x}=\frac{\pi d^{2}}{4}\left[A Y N_{1}+B \rho V^{2} N_{2}+(C \sqrt{\rho Y} \cdot V+D \rho d \cdot \dot{V}) N_{3}\right]
$$

where $N_{1}, N_{2}$ and $N_{3}$ are three dimensionless parameters relating to the nose shape and friction effect. $N_{1}$ and $N_{2}$ are employed in the impact function $I$ and the geometry function $N$ of projectile, respectively. $N_{3}$ is employed in the term of dummy mass, which will be discussed in Section 4. Various expressions of $N_{1}, N_{2}$ and $N_{3}$ of the common convex nose shape and various typical noses of projectiles, e.g., ogive, conical and blunt noses, are formulated in Chen and $\mathrm{Li}$ [2], $\mathrm{Li}$ and Chen [3] and Chen et al [9], respectively. In general, the mathematical definitions of $N_{1}, N_{2}$ and $N_{3}$, avoid the ambiguous description of the nose shape of projectiles in different empirical formulae.

\section{The third dimensionless number and DOP}

Using Newton's second law, eqn (2) can be re-written as

$$
-M_{m} \dot{V}=-M_{m} V \frac{\mathrm{d} V}{\mathrm{~d} x}=\frac{\pi d^{2}}{4} A Y N_{1}\left(1+\frac{B N_{2}}{A N_{1}} \frac{\rho V^{2}}{Y}+\frac{C N_{3}}{A N_{1}} \sqrt{\frac{\rho V^{2}}{Y}}\right)
$$

After accounting for the dummy mass, the projectile mass $M$ is modified as

$$
M_{m}=\left(M+\frac{\pi \rho d^{3}}{4} D N_{3}\right)
$$


In contrast to Chen and $\mathrm{Li}$ [2] and $\mathrm{Li}$ and Chen [3], a third term is included in the right-hand side of eqn (3).

A dimensionless number is expressed as

$$
\xi=\frac{C^{2} N_{3}^{2}}{4 A B N_{1} N_{2}}=\frac{C^{2}}{4 A B}
$$

which is related to the damping term of eqn (2), and hence $\xi$ is defined as the dimensionless damping function. According to the definitions of nose parameters $N_{1}, N_{2}$ and $N_{3}$ in Chen and $\mathrm{Li}$ [2], Li and Chen [3] and Chen et al [9], we have $N_{3}^{2} \cong N_{1} N_{2}$. The above equation is achieved exactly without any sliding friction accounted for. Thus we may conclude that $\xi$ is independent of projectile geometry and only relates to the material behaviour of targets.

The dimensionless depth of penetration may be integrated from eqn (3) under the initial condition $V=V_{0}$,

$$
\frac{X}{d}=\frac{2}{\pi} N\left\{\ln \left(1+2 \sqrt{\xi \frac{I}{N}}+\frac{I}{N}\right)-\int_{\sqrt{\xi}}^{\sqrt{I / N}+\sqrt{\xi}} \frac{2 \sqrt{\xi} d \bar{U}}{\left[\bar{U}^{2}+(1-\xi)\right]}\right\}
$$

where $\bar{U}$ is only a process parameter in the integral process. The dimensionless impact function $I$ and the geometry function of projectile $N$ are still employed in the formulation $[2,3]$

$$
I=\frac{I^{*}}{A N_{1}}, N=\frac{\lambda}{B N_{2}}
$$

where dimensionless impact factor $I^{*}$ and mass ratio $\lambda$ are modified as

$$
I^{*}=\frac{M_{m} V_{0}^{2}}{d^{3} Y}, \lambda=\frac{M_{m}}{\rho d^{3}}
$$

The effect of the impact function $I$ and geometry function $N$ on penetration has been discussed in detail previously and will not be repeated here.

Eqn (6) indicates that only three dimensionless numbers, i.e. $I, N$ and $\xi$, are dominant parameters to determine the dimensionless final depth of penetration into a target impacted by a rigid projectile. $\xi$ is the third dimensionless number besides $I$ and $N$ in the penetration dynamics of rigid projectiles.

Furthermore, referring to the dynamic cavity expansion theory, commonly we have $A>B, A>C$ and $B \approx C$. It rationally induces that $0 \leq \xi<1$. If not considering the damping term, i.e., $C=0$ or $\xi=0$, the dimensionless DOP is,

$$
\frac{X}{d}=\frac{2}{\pi} N \cdot \operatorname{In}\left(1+\frac{I}{N}\right)
$$

which is exactly the same as that in Chen and $\mathrm{Li}$ [2] and Li and Chen [3].

If $0<\xi<1$, i.e., $(1-\xi)>0$, eqn (6) is simplified as

$$
\frac{X}{d}=\frac{2}{\pi} N\left\{\ln \left(1+2 \sqrt{\xi \frac{I}{N}}+\frac{I}{N}\right)-\frac{2 \sqrt{\xi}}{\sqrt{1-\xi}}\left[\tan ^{-1} \frac{\sqrt{I / N}+\sqrt{\xi}}{\sqrt{1-\xi}}-\tan ^{-1} \frac{\sqrt{\xi}}{\sqrt{1-\xi}}\right]\right\}
$$




\section{Perforation of thick plates by a sharp-nosed projectile}

The normal perforation of thick metallic plates by a sharp-nose rigid projectile is further studied based on the general penetration resistance. For a projectile with a sharp nose, the plugging stage is usually omitted and only the penetration dominates in the whole perforation process. Perforation occurs when the nose tip of the projectile reaches the rear surface of the target. We define $\chi=H / d$ as the dimensionless thickness of the plate and $H$ as the target thickness. The residual or exit velocity of the projectile is assumed to be $V_{r}$. The boundary effect of the rear free surface is negligible, eqn (10) is re-written as

$$
\chi=\frac{2}{\pi} N\left\{\ln \left(\frac{1+2 \sqrt{\xi \frac{I}{N}}+\frac{I}{N}}{1+2 \sqrt{\xi \frac{I_{\mathrm{r}}}{N}}+\frac{I_{\mathrm{r}}}{N}}\right)-\frac{2 \sqrt{\xi}}{\sqrt{1-\xi}}\left[\tan ^{-1} \frac{\sqrt{\frac{I}{N}}+\sqrt{\xi}}{\sqrt{1-\xi}}-\tan ^{-1} \frac{\sqrt{\frac{I_{\mathrm{r}}}{N}}+\sqrt{\xi}}{\sqrt{1-\xi}}\right]\right\}
$$

where $I_{r}=\frac{M_{m} V_{r}^{2}}{A N_{1} d^{3} Y}$ is the impact function corresponding to the residual velocity $V_{r}$.

The ballistic limit is achieved at critical perforation with zero residual/exit velocity, i.e., $V_{r}=0$. In that case, we have,

$$
\chi=\frac{2}{\pi} N\left\{\ln \left(1+2 \sqrt{\xi \frac{I_{B L}}{N}}+\frac{I_{B L}}{N}\right)-\frac{2 \sqrt{\xi}}{\sqrt{1-\xi}}\left[\tan ^{-1} \frac{\sqrt{I_{B L} / N}+\sqrt{\xi}}{\sqrt{1-\xi}}-\tan ^{-1} \frac{\sqrt{\xi}}{\sqrt{1-\xi}}\right]\right\}
$$

where $I_{B L}=\frac{M_{m} V_{B L}^{2}}{A N_{1} d^{3} Y}$ corresponds to the ballistic limit $V_{B L}$. In general, the ballistic limit $V_{B L}$ and residual velocity $V_{r}$ may be solved with eqns (11) and (12), respectively. Some special simplified cases for the engineering application will be given in the next section.

\section{Special cases of perforation}

\section{$5.1 \xi=0$}

Without considering the damping effect in the perforation process, the simplified ballistic limit and residual velocity are obtained from eqns (11) and (12), respectively, when $\xi=0$, which have the same forms as those in Chen and $\mathrm{Li}$ $[2]$.

$$
\begin{gathered}
V_{\mathrm{BL}}^{2}=\frac{A N_{1} Y}{B N_{2} \rho}\left[\exp \left(\frac{\pi \chi}{2 N}\right)-1\right] \\
V_{\mathrm{r}}=\sqrt{\frac{\left(V_{0}^{2}-V_{\mathrm{BL}}^{2}\right)}{\exp \left(\frac{\pi \chi}{2 N}\right)}}
\end{gathered}
$$




\section{$5.2 I / N \rightarrow 0$}

Usually a sharp nose projectile has a large value of geometry function $N$, while it perforates a metallic plate at a relative low impact velocity, i.e., with a small impact function $I$. Thus, we have $I / N \rightarrow 0$ [2]. It indicates that the value of the damping function $\xi$ is smaller for most of the experimental results in the next section, i.e., $\xi<0.1$. It may be further simplified as,

$$
\begin{gathered}
V_{B L}=\sqrt{\frac{A N_{1} Y}{B N_{2} \rho}}\left[\frac{\xi}{1-\xi} \sqrt{\xi}+\sqrt{\frac{\pi \chi}{2 N}}\right] \\
{\left[\frac{I_{r}}{N}-\frac{2 \xi \sqrt{\xi}}{(1-\xi)} \sqrt{\frac{I_{r}}{N}}\right]=\left[\frac{I}{N}-\frac{2 \xi \sqrt{\xi}}{(1-\xi)} \sqrt{\frac{I}{N}}\right]-\left[\frac{I_{B L}}{N}-\frac{2 \xi \sqrt{\xi}}{(1-\xi)} \sqrt{\frac{I_{B L}}{N}}\right]}
\end{gathered}
$$

If $\xi<0.01$, the effect of the damping function $\xi$ is regarded as completely ignored, and much simpler formulae can be conducted from eqns (15) and (16)

$$
\begin{aligned}
V_{B L} & =\sqrt{\frac{\pi \chi A N_{1} Y}{2 \lambda \rho}} \\
V_{\mathrm{r}} & =\sqrt{V_{0}^{2}-V_{\mathrm{BL}}^{2}}
\end{aligned}
$$

\section{Experimental analysis}

\subsection{Deep penetration in metal and concrete targets}

According to the definition of three dimensionless numbers, all the test data [11-15] and theoretical predictions are re-grouped. In view of strain hardening and strain-rate sensitivity, the value of $C(C=0.94)$ for 6061-T6511 aluminium was pioneered by Warren and Forrestal [16] with the introduction of a once power of velocity. 0.94 is regarded as the approximate value of $\mathrm{C}$ for all the aluminium plates used in the present manuscript, which was cited from different references.

Generally, the test data of DOP are a little smaller than the theoretical predictions of Chen and $\mathrm{Li}$ [2], which only depend on $I$ and $N$. However, it is a little larger than the present analysis accompanied with $\xi$, especially in the range of higher velocity impacts. Fig. 2 presents the variation of dimensionless DOP $X / d$ with $I$ for different values of $N$. In spite of the great difference in projectile geometry, target material and impact velocity, the test data are well represented in Fig. 2. When $I$ is large enough, the prediction values for the dimensionless DOP are smaller than the test data.

Figs. 3-5 compare the theoretical predictions with test data for the 6061-T6511 aluminium target by ogive, spherical and conical nose projectiles, respectively. Fig. 6 shows both the predictions and test data of penetration into concrete targets by ogive-nose projectiles [8]. Obviously, the predictions of [2] and the present analysis are the upper and lower envelopes of test data, respectively. 


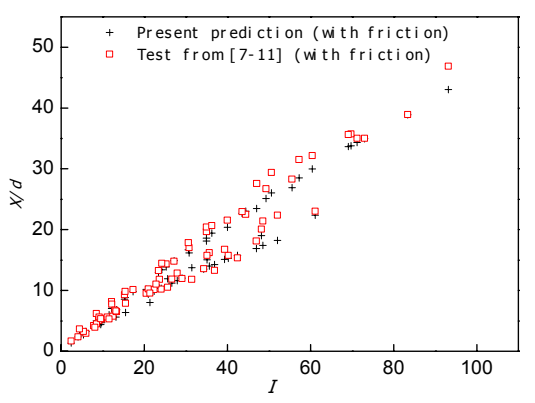

Figure 2: Dependence of DOP on $I$ for various aluminum alloy targets.

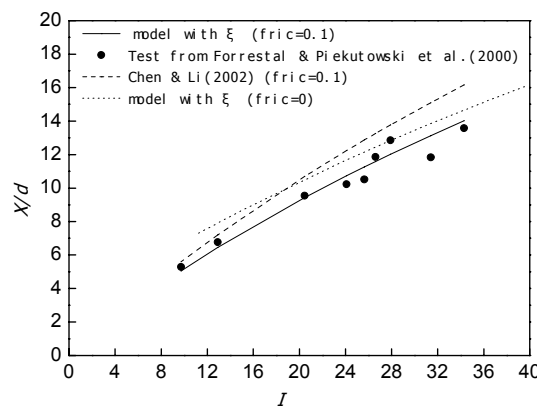

Figure 4: Dependence of DOP on $I$ for spherical projectile penetrating aluminum.

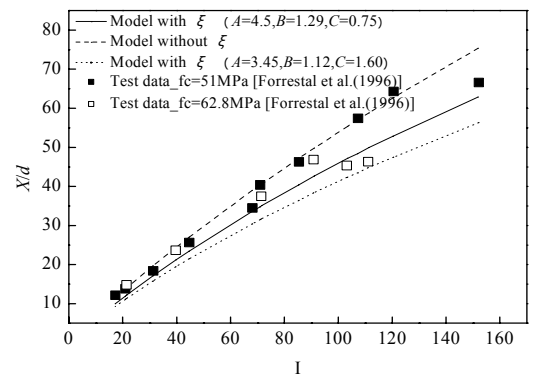

Figure 6: Dependence of DOP on $I$ for ogive projectile penetrating aluminium.

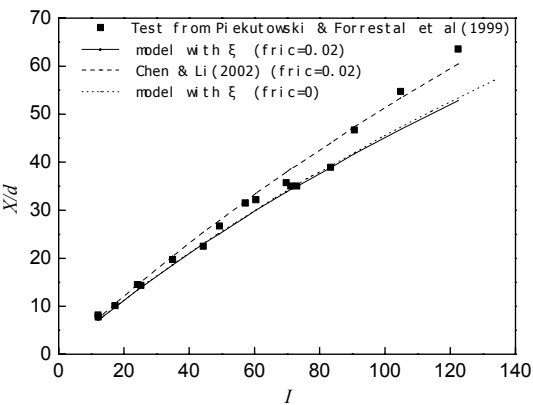

Figure 3: Dependence of DOP on $I$ for ogive projectile penetrating aluminium.

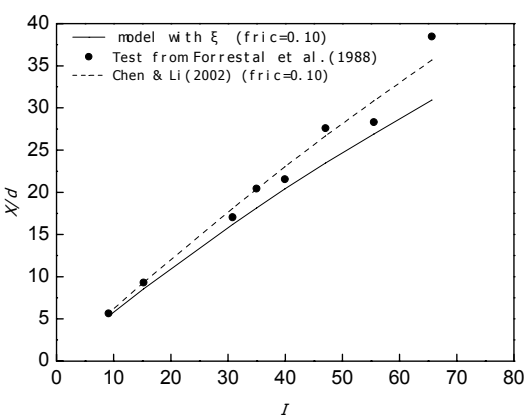

Figure 5: Dependence of DOP on $I$ for conical projectile penetrating aluminium.

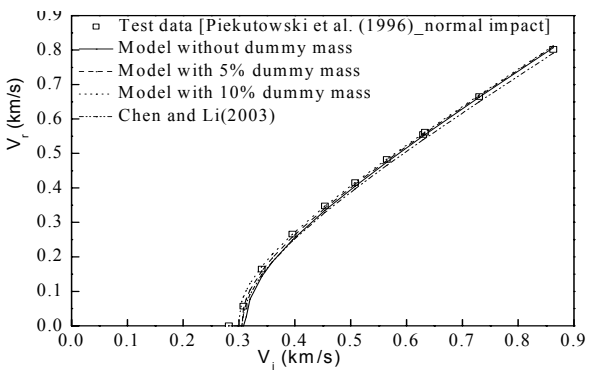

Figure 7: $\quad$ Prediction of ballistic performance and test data. 


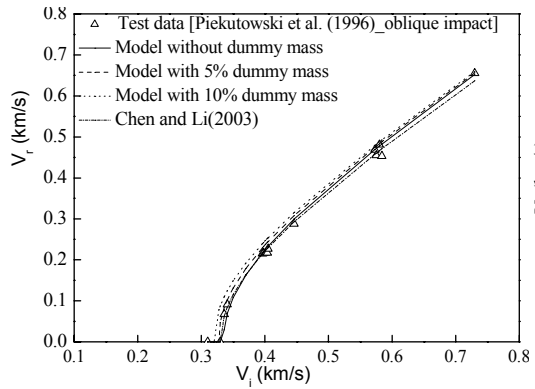

Figure 8: $\quad$ Prediction of ballistic performance and test data.

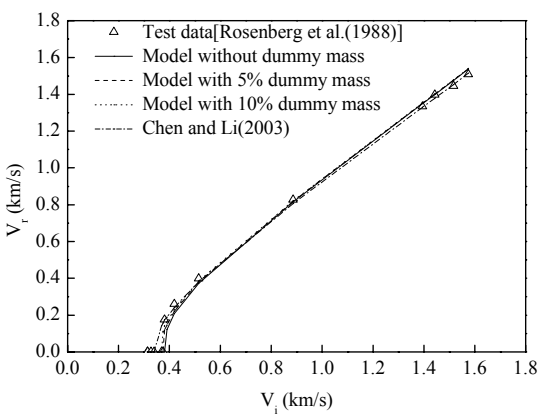

Figure 10: Prediction of ballistic performance and test data.

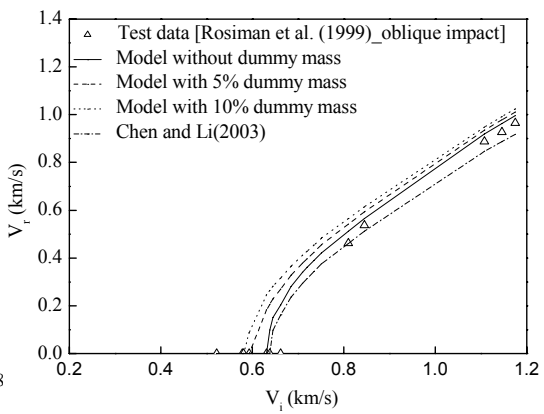

Figure 9: $\quad$ Prediction of ballistic performance and test data.

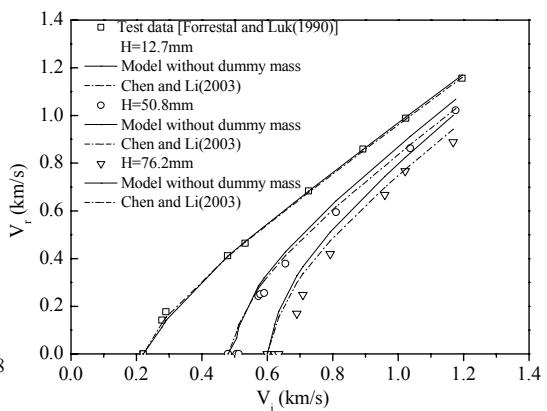

Figure 11: $\quad$ Prediction of ballistic performance and test data.

As shown in Figs. 2-6, the deviation of the present model after introducing $\xi$ from Chen and $\mathrm{Li}$ [2] never exceeds $15 \%$. It clearly indicates that $\xi$ has less influence on penetration capability than $I$ and $N$. Among the three dimensionless numbers, the impact function $I$ is the most sensitive, and the geometry function of projectile $N$ takes second place. The smaller the value of $N$, the more notable the influence of $N$ on DOP. However, when $N$ is large enough, e.g., $N>100, N$ is never sensitive to DOP. Whether shallow or deep penetration, the influence of $\xi$ on the penetration capability is consistent and smaller.

\subsection{Perforation of metallic plates}

In this section, all the experimental data in [17-20] and theoretical predictions are re-grouped. Figs. 7-11 show the experimental results and corresponding theoretical predictions. Obviously, it improves the target resistance against 
penetration by introducing damping function $\xi$, and thus, the ballistic limit increases while the residual velocity decreases a little in comparison to that in Chen and $\mathrm{Li}$ [2]. However, after accounting for the dummy mass, the ballistic limit may decrease and even become smaller than the prediction of Chen and $\mathrm{Li}$ [2], while being closer to the test results. The dummy mass is beneficial for improving the ballistic performance of the projectile. In general, the damping function $\xi$ conflicts with the dummy mass $M_{m}$ and they compensate for each other in a penetration. A theoretical prediction that takes into account $\xi$ and $M_{m}$ simultaneously may become more appropriate in practice.

Through fitting the test data, we may evaluate the influence of the dummy mass term on penetration/perforation reversely. As shown in Figs. 7-11, accompanied with the damping effect, the residual velocity and ballistic limit lead to good predictions of experimental results if the mass of the projectile increases by $5 \%-10 \%$. In other words, the dummy mass may contribute to the mass of the projectile from $5 \%$ to $10 \%$. Thus the value of $D$ can be deduced by $M_{\mathrm{m}}=\left(M+\pi \rho d^{3} / 4 \cdot D N_{3}\right)$ and varies between 1 and 2 .

\section{Conclusions}

In this paper, a third dimensionless parameter, i.e., the damping function $\xi$, is defined in the penetration dynamics of rigid projectiles besides the impact function $I$ and the geometry function of projectile $N$ introduced previously. They dominate in the penetration of rigid projectiles. The dimensionless formula of DOP is conducted in expression of $I, N$ and $\xi$ for general convex shapes of various rigid projectiles. Considering the viscous effect and dummy mass of the projectile, the normal perforation of thick metallic plates by sharp nose rigid projectiles are studied further and only $I, N$ and $\xi$ as well as the dimensionless target thickness $\chi$ dominate in perforation. Theoretical predictions of penetration and perforation agree quite well with the individual test data of different projectiles and impact velocities as well as different targets.

\section{Acknowledgement}

This project is funded by the National Natural Science Foundation of China under Contract No. 10672152.

\section{References}

[1] Poncelet J.V., Cours de Mecanique Industrielle, Paris, 1835.

[2] Chen X.W, Li Q.M. Deep penetration of a non-deformable projectile with different geometrical characteristics. Int J Impact Eng 2002, 27:619-37.

[3] Li Q.M., Chen X.W. Dimensionless formulae for penetration depth of concrete target impacted by a non-deformable projectile. Int J Impact Eng 2003, 28(1): 93-116. 
[4] Forrestal M.J., etc. A model to estimate forces on conical penetrators into dry porous rock. J Appl Mech, 1981;18: 25-29.

[5] Forrestal M.J., etc. Penetration into targets described by locked hydrostats and strength. Int J Solids Struct 1981;17(9): 915-24.

[6] Forrestal M.J., Luk V.K. Dynamic spherical cavity-expansion in a compressible elastic-plastic solid. ASME J Appl Mech 1988;55:275-9.

[7] Forrestal M.J, Luk V.K. Penetration into soil targets. Int J Impact Engng 1992, 12:427-44

[8] Forrestal M.J, Frew D.J., Hanchak S.J., Brar N.S. Penetration of grout and concrete targets with ogive-nose steel projectiles. Int J Impact Engng 1996, 18(5): 465-76

[9] Chen X.W., Li X.L., Huang F.L., Wu H.J., Chen Y.Z. Damping Function in the Penetration/Perforation Struck by Rigid Projectiles. Int J Impact Engng, 2007, doi:10.1016/j.ijimpeng.2007.07.011.

[10] Chen X.W. Dynamics of Metallic and Reinforced Concrete Targets Subjected to Projectile Impact. PhD Thesis, Nanyang Technological University, Singapore, 2003.

[11] Forrestal M.J, Okajima K., Luk V.K. Penetration of 6061-T651 aluminum targets with rigid long rods. Trans ASME J Appl Mech 1988, 55:755-60

[12] Forrestal M.J, Brar N.S, Luk V.K. Penetration of strain-hardening targets with rigid spherical-nose long rods. Trans ASME J Appl Mech 1991, 58(1): $7-10$

[13] Forrestal M.J., Luk V.K. Penetration of 7075-T651 aluminum targets with ogival-nose rods. Int J Solids Struct 1992, 29:1729-36

[14] Piekutowski A.J., Forrestal M.J., Poormon K.L., Warren T.L. Penetration of 6061-T6511 aluminum targets by ogival-nose steel projectiles with striking velocities between 0.5 and $3.0 \mathrm{~km} / \mathrm{s}$. Int J Impact Engng 1999, 23:723-34

[15] Forrestal M.J., Piekutowski A.J. Penetration experiments with 6061-T6511 aluminum targets and spherical-nose steel projectiles at striking velocities between 0.5 and $3.0 \mathrm{~km} / \mathrm{s}$. Int J Impact Engng 2000, 24:57-67

[16] Warren T.L., Forrestal M.J. Effects of strain hardening and strain-rate sensitivity on the penetration of aluminum targets with spherical-nose rods. Int. J. Solids Struct 1998; 35:3737-52

[17] Rosenberg Z., Forrestal M.J. Perforation of aluminium plates with conicalnosed rods-additional data and discussion. J Appl Mech Trans ASME 1988; 55:236-8

[18] Forrestal M.J., Luk V.K. Perforation of aluminium armor plates with conical-nose projectiles. Mech Mater 1990; 10:97-105

[19] Piekutowski A.J., Forrestal M.J., Poormon K.L., Warren T.L. Perforation of aluminum plates with ogive-nose steel rods at normal and oblique impacts. Int J Impact Engng 1996;18(7-8):877-87

[20] Roisman I.V., Weber K., Yarin A.l., Hohler V., Rubin M.B. Oblique penetration of a rigid projectile into a thick elastic-plastic target: theory and experiment. Int J Impact Engng 1999; 22:707-26 